Optimizing asphalt mix design process using artificial neural network and genetic algorithm

\title{
Optimizing asphalt mix design process using artificial neural network and genetic algorithm
}

\author{
Haissam Sebaaly \\ Department of Civil Engineering, University of Pretoria, Private Bag X20, Hatfield 0028, South Africa \\ E-mail: haissam16@hotmail.com \\ Sudhir Varma \\ Former Graduate Research Assistant, Department of Civil and Environmental Engineering, Michigan State \\ University, Engineering Building 428, S. Shaw Lane, Room 3546, \\ East Lansing, MI 48824 \\ E-mail: varmasud@msu.edu \\ James W. Maina ${ }^{1}$ \\ Department of Civil Engineering, University of Pretoria, Private Bag X20, Hatfield 0028, South Africa \\ E-mail: james.maina@up.ac.za
}

\section{Highlights}

- Conventional asphalt mix design is a time consuming iterative process requiring significant amount of materials.

- Significant information is available in a database on historical mix designs.

- This paper presents a procedure that was followed to successfully develop Artificial Neural Network (ANN) and Genetic Algorithm (GA) models that utilized the database and automate selection of aggregate gradation and binder content to produce asphalt mixtures that comply with applicable specification requirements.

\footnotetext{
${ }^{1}$ Corresponding author.
} 
Abstract: Selection of aggregate gradation and binder content for asphalt mix design, which comply with specification requirements, is a lengthy trial and error procedure. Success in performing mix design rely largely on experience of the designer. This paper presents development of an automatic mix design process with the ability to both predict and optimize asphalt mix constituents to obtain desired mix properties. A successful automatic process requires the use of local past experience translated into a design aid tool, which then predicts properties of asphalt mix without actually testing the mix in laboratory. In the proposed approach, simple multilayer perceptron structure Artificial Neural Network (ANN) models were developed using 444 Marshall mix design data. The ANN models were able to predict both air voids and theoretical maximum specific gravity of asphalt mix to within $\pm 0.5 \%$ and \pm 0.025 , respectively, for $99.6 \%$ of the time. After that, the ANN models were called by a non-linear constrained genetic algorithm to optimize asphalt mix, while satisfying the Marshall requirements defined in the formulation as constraints. Durability of the optimized mix is ensured by introducing a constraint on adequacy of asphalt film thickness. The developed mix design aid tool is compiled into a computer software called Asphalt Mix Optimization (AMO) that can be used by road agencies as a mix design tool. A case study is presented to demonstrate the ability of the model to optimize aggregate gradation and minimize binder content in asphalt mix. The computed ANN outputs and the optimized gradation were found to compare well with laboratory measured values. Although, Marshall compacted mixes were used in demonstrating the approach, this method is general and can be applied to any mix design procedure.

Keywords: Artificial neural network, genetic algorithm, gradation, asphalt mix design, optimization 


\section{INTRODUCTION}

Asphalt mix design entails proportioning of aggregates and binder in a design recipe to obtain desirable mechanical and volumetric properties for the mixture produced. The mechanical and volumetric properties significantly influence the performance and durability of asphalt mix. Influences of aggregate on properties of bituminous mix are well known and their requirements are specified by client bodies around the world. The initial efforts in finding optimal gradation were based on the principle that maximizing the density would result in a denser gradation, leading to a better performing mix (Fuller and Thompson, 1907; Roberts, et al., 1996). However, it has been reported in several studies that the maximum density results in low voids in Mineral Aggregate (VMA) and, therefore, low binder content $\left(\mathrm{P}_{\mathrm{b}}\right)$ and Air Void (Va). McLeod (1956) proposed to use volumetric relationship in asphalt mix design, instead of following maximum density.

The effect of aggregate gradation on volumetric parameters, strength and permanent deformation in asphalt mixes have been extensively studied by various researchers. Researchers have stressed on the importance of packing characteristics of aggregate gradation based on relative volume of different parts of gradation (Birgisson and Ruth, 2001), (Vavrik et al., 2002), (Roque et al., 2006). The approach is based on the fact that change in packing of coarse and fine aggregate directly relate to aggregate interlocking and void in aggregate in compacted asphalt mix.

Although the current gradation design methods provide some indications on volumetric and mechanical properties, essentially they are empirical and require a trial and error process to establish an optimum blend. Further, the methods may be useful in comparing different gradations but cannot accurately predict volumetric and mechanical properties of asphalt mixes 
without testing them in laboratory. Recently, in an effort to accurately estimate volumetric and mechanical properties of asphalt mixes, some researchers have adopted more computational approach, which considers particle to particle interaction (Shen and $\mathrm{Yu}, 2011)$, (Li and Wang, 2015). These approaches are based on discrete element method, which uses force functions to define particle to particle interaction. Limitation of such models is the significant increase in the computational effort when smaller particles $(<1.18 \mathrm{~mm})$ are included in the analysis. Moreover, the ability of these models to reach equilibrium is often significantly reduced on including the small particles in the analysis.

It is evident that selection of aggregate gradation for asphalt mix design, which would comply with volumetric and mechanical requirement is complex and rely mainly on experience. Random selection based on trial and error to obtain an optimum gradation is nearly impossible. This is because asphalt mix formulation depends on several factors such as specific gravity, texture, shape and absorption properties of locally used aggregates and binder. Therefore, it is important that the local past experience be translated into a design aid tool. Ozturk and Kutay (2014) used laboratory data collected from 1,817 Superpave mix designs to develop a novel Artificial Neural Network (ANN) model ANN-AM. The objective of their study was to use mix design data in training ANN which can then be used as a virtual Superpave mix design tool. Aggregate gradation, specific gravity, $\mathrm{PG}$ grade, $\mathrm{P}_{\mathrm{b}}$, initial, design and maximum gyrations were used as input in the model to predict Va, VMA and Voids Filled with Bitumen (VFB) at different gyration levels. The objective of the current study is to first train an ANN model for Marshall mix design and then demonstrate that such a model can be used to develop an automated scheme to obtain optimized asphalt mix with desire properties. 
In recent years, artificial intelligence tools have gained traction to learn surrounding conditions and provide successful responses. In pavement engineering, ANN has been used to interpret complex data obtained from field, laboratory or computer simulations. ANN has mostly been applied in three major areas in pavement engineering, i.e. evaluation of structural condition of pavement, forecasting distress condition of pavement and estimation of asphalt mix properties. In structural evaluation, ANN has been mainly used to interpret Falling Weight Deflectometer (FWD) data through an inverse scheme to predict modulus value of pavement layers (Gopalakrishnan et al., 2014), (Li and Wang, 2017). Apart from estimating deflections for FWD analysis, researchers have also demonstrated application of ANN in estimating stresses, layer thickness and joint load transfer in pavement. In function evaluation, $\mathrm{ANN}$ has been used to predict deterioration of pavement performance in terms of roughness, rut depth and cracking (Huang and Moore, 1997), (Choi et al., 2004), (Yang et al., 2003), (Lytton et al., 2010). ANN has also been used for crack detection, classification and determination of severity. In asphalt mix evaluation, ANN has been successfully used to estimate asphalt mix properties such as indirect tensile strength, density and dynamic modulus (Krcmarik et al., 2016), (Commuri et al., 2011), (Far et al., 2009).

\section{Objective}

The goal of the present study is to develop an automatic design tool, which uses local experience of road agencies to aid in optimizing asphalt mix. Therefore, the procedure followed was (i) to obtain mix properties from construction projects and use them in developing ANN models and (ii) develop an optimization scheme using Genetic Algorithm and automate mix design procedure.

Although Superpave has gained prominence as the state-of-the-art method for asphalt mix design, there are still many road agencies around the world where Marshall mix design is 
Optimizing asphalt mix design process using artificial neural network and genetic algorithm

still the most prevalent asphalt mix design method. Therefore, in this study Marshall mix design data for mixes intended to be used for pavement construction projects were collected and used in developing the model. Another advantage of the developed model is that it would be helpful for agencies that have experience with Marshall mix performance, but plan to move towards Superpave mix design. In such cases, the ANN models can assist the designer to predict Marshall Properties for Superpave design mixes that have aggregate and binder properties that are within the limits of the databased used in developing the ANN models.

Based on the existing literature and despite successful demonstration of possible advantageous ANN implementation in pavement engineering, it has not been adopted in practice. The main obstacles in adopting ANN in practice is lack of background information and complex architecture of ANN models. Therefore, one of the objective of this study is to develop ANN model that uses simple architecture and readily available asphalt mix properties as inputs.

\section{RESEARCH APPROACH AND DATABASE}

A model that is capable of satisfactorily estimating volumetric properties through simple input parameters can be used to conduct a virtual mix design. Further, the model can then be called by an optimization scheme to produce mixes with optimized characteristics (objectives). This is because the model would be able to evaluate different asphalt mix scenarios generated by varying the model inputs.

Although this paper presents models developed for Marshall mix design, the approach described here is general and can be applied to any other mix design method. In Marshall mix design, four asphalt mix parameters, i.e. stability, flow, bulk specific gravity $\left(\mathrm{G}_{\mathrm{mb}}\right)$ and theoretical maximum specific gravity $\left(\mathrm{G}_{\mathrm{mm}}\right)$ are measured in laboratory and used in further analysis for acceptance or rejection of the mix. Initially, this study considered these four mix 
Optimizing asphalt mix design process using artificial neural network and genetic algorithm

design parameters as the target variables. However, it was later realized that a much better ANN model for Va can be developed compared to $G_{m b}$. Finally, it was decided to use Va as a target variable instead of $G_{m b}$ and then Va along with $G_{m m}$ were used to calculate $G_{m b}$. Therefore, four separate ANN models were developed to predict the four targeted variables. The input variables used in all the four models were the same, which were obtained from the 444 Marshall Mix data.

In subtropical hot desert climate regions like the one in the present study, bleeding is one of the major concern. Therefore, there is a natural tendency in the pavement industry in regions with such climate to use lower binder content. Selection of lower binder content to avoid any potential bleeding may jeopardize durability of asphalt mixes in these regions. Studies have shown that durability is directly related to bitumen film thickness $\left(\mathrm{FT}_{\mathrm{b}}\right)$. Therefore, to ensure that sufficient durability is achieved in the mix, in addition to Marshall mix design parameters, minimum $\mathrm{FT}_{\mathrm{b}}$ requirement is also included in the model.

\section{Mix design ANN database}

All the input and the targeted variables in the ANN models were from laboratory conducted Marshall mix designs for actual pavement construction projects. A total of 444 Marshall mix design data were used in the study to train, verify and test the ANN models. The Marshall mixes used in this study were compacted at 75 number of blows per face of specimen. The mixes in the database are produced using two grade of binders, PG 64-10 and PG 76-10. The binder PG 64-10 is unmodified asphalt of Pen grade 60/70 expected to be from a single source. The binder PG 7610 is modified asphalt obtained from four different sources. All the aggregates were crushed gabbro, expected to be from ten different suppliers. Therefore, the mix design database included two types of binder and a single type of aggregate. 
The temperatures of compaction and mixing were obtained from binder's viscosity temperature chart. For modified bitumen, mixing temperature corresponds to a viscosity range of $0.75 \pm 0.05$ Pas and compaction temperature corresponds a viscosity range of 1.4 \pm 0.1 Pas, measured at $20 \mathrm{rpm}$ with Brookfield Spindle No. 27. For unmodified bitumen the temperature at a rotational viscosity of $0.17 \pm 0.02$ Pas was used for mixing and $0.28 \pm 0.03$ Pas was used for compaction.

The targeted variables in this study are stability, flow, $V a$ and $G_{m m}$. Here, the independent variables are those factors that can be obtained from simple tests on aggregates and binder in the laboratory, before a mix is produced. The independent variables identified in this study are binder content, $\mathrm{G}_{\mathrm{sb}}$ of combine gradation, higher $\mathrm{PG}$ of the binder and percentage passing the $0.075 \mathrm{~mm}, 0.425 \mathrm{~mm}, 1.18 \mathrm{~mm}, 2.36 \mathrm{~mm}, 4.75 \mathrm{~mm}, 9.5 \mathrm{~mm}, 12.5 \mathrm{~mm}, 19.0 \mathrm{~mm}$ and $25.0 \mathrm{~mm}$ sieves. Table 1 shows the maximum, minimum, average and standard deviation of each variable in the database. The database comprises of Marshall test results conducted at different binder contents of each mix design.

TABLE 1: Range of variables used in developing ANN model

\begin{tabular}{|c|c|c|c|c|c|c|}
\hline \multicolumn{3}{|c|}{ Variables } & Minimum & Maximum & Average & $\begin{array}{l}\text { Standard } \\
\text { deviation }\end{array}$ \\
\hline \multirow{12}{*}{$\begin{array}{c}\text { Input } \\
\text { variables }\end{array}$} & \multirow{9}{*}{$\begin{array}{c}\text { Percent } \\
\text { passing } \\
\text { sieve } \\
\text { size }\end{array}$} & $25 \mathrm{~mm}$ & 91 & 100 & 99 & 2.0 \\
\hline & & $19 \mathrm{~mm}$ & 78 & 100 & 97 & 5.3 \\
\hline & & $12.5 \mathrm{~mm}$ & 64 & 87 & 79 & 4.7 \\
\hline & & $9.5 \mathrm{~mm}$ & 50 & 76 & 68 & 4.4 \\
\hline & & $4.75 \mathrm{~mm}$ & 39 & 54 & 47 & 3.5 \\
\hline & & $2.36 \mathrm{~mm}$ & 27 & 42 & 32 & 2.4 \\
\hline & & $1.18 \mathrm{~mm}$ & 10 & 31 & 21 & 2.7 \\
\hline & & $0.425 \mathrm{~mm}$ & 7 & 19 & 12 & 1.8 \\
\hline & & $0.075 \mathrm{~mm}$ & 3.0 & 6.1 & 4.2 & 0.4 \\
\hline & \multicolumn{2}{|c|}{$\mathrm{Pb}_{\mathrm{b}} \%$} & 2.5 & 5.5 & 4.1 & 0.7 \\
\hline & \multicolumn{2}{|c|}{$\mathrm{G}_{\mathrm{sb}}$} & 2.824 & 2.972 & 2.889 & 0.039 \\
\hline & \multicolumn{2}{|c|}{$\begin{array}{c}\text { PG high } \\
\text { temperature }{ }^{\circ} \mathrm{C}\end{array}$} & 64 & 76 & na & na \\
\hline \multirow{4}{*}{$\begin{array}{c}\text { Target/ } \\
\text { output } \\
\text { variables }\end{array}$} & \multicolumn{2}{|c|}{$\mathrm{G}_{\mathrm{mm}}$} & 2.619 & 2.841 & 2.719 & 0.048 \\
\hline & \multicolumn{2}{|c|}{$\mathrm{Va} \%$} & 1.1 & 11.6 & 5.8 & 2.0 \\
\hline & \multicolumn{2}{|c|}{ Stability, kN } & 9.0 & 31.7 & 15.8 & 3.8 \\
\hline & \multicolumn{2}{|c|}{ Flow $(0.25 \mathrm{~mm})$} & 2.0 & 4.9 & 3.1 & 0.5 \\
\hline
\end{tabular}


Two main limitations of ANN based models are: (1) the quality of prediction depends on the quality of ANN trained and (2) performance of ANN models is highly related to the data used in the development of the model. In fact, only the problems which are within the bounds of the data set considered in the training of ANN are expected to produce reasonable solution. Therefore, since the ANN models were developed using the data space shown in Table 1, it is expected that the model would be able to perform well within these variable ranges.

\section{PROPOSED GRADATION SELECTION PROCEDURE}

The ability to predict asphalt mix properties and the ability to optimize gradation to obtain desired mix properties are both important to automate asphalt mix design process. Therefore, the primary component in the aggregate gradation selection procedure proposed in the present study is the ANN model that can predict asphalt mix properties. The inputs to this ANN model are the candidate solutions from non-linear constrained Genetic Algorithm (GA).

In practice, hot bin aggregates that are intended to be used in asphalt production are tested in laboratory. To obtain a combined gradation, the hot bin aggregates are blended in certain percentages by weight. The combined gradation is then mixed with certain percentage of binder to produce asphalt mix. Needless to say that, the selected hot bin and binder percentages shall be such that the mix provides desirable volumetric and mechanical properties which is the ultimate objective of mix design. Therefore, in the proposed approach, percentage of hot bins and percent of binder are considered as the unknowns to be searched. As explained later, the non-linear constrained GA systematically varies these unknowns to reach an optimized gradation. It is assumed that specific gravity of the individual hot bins and PG grade of the binder that are intended to be used in asphalt mix production are known. The specific gravity of 
the combined gradation is calculated from the specific gravity of individual hot bins using the equation from the Asphalt Institute MS-2 (Asphalt Institute, 2014)

$$
G_{s b}=\frac{1}{\frac{P_{1}}{100 G_{s b 1}}+\frac{P_{2}}{100 G_{s b 2}}+\cdots \frac{P_{n}}{100 G_{s b n}}}
$$

where, $P_{i}$ is the percentage (by weight) of hot bin $i$ in the combined gradation, $G_{s b i}$ is the specific gravity of hot bin $i$ and $n$ is the total number of hot bins.

The independent variables listed in Table 1 are percent binder content, percent passing individual sieve sizes (calculated from trial hot bin percentages), specific gravity of combine gradation (calculated using Equation 1) and PG grade high temperature are used in ANN model to predict target variables.

\section{ANN models to predict asphalt mix design parameters}

Analogous to the interconnected neurons in humans that process and transmits information, ANNs consist of interconnected set of nodes (artificial neurons). In ANN, nodes are essentially mathematical functions, which receive weighted inputs. As shown in Figure 1, after processing the inputs, the nodes pass output to other nodes in the next layer (if exists) through weighted links. ANNs learn to predict outputs by adjusting these weights.

A large class of different ANNs are possible based on the number of nodes, arrangement of nodes, transfer/activation function of nodes and training function of neural network. Multilayer Perceptron (MLP) is one of the most used ANN for function approximation and is also used here. MLP consists of at least three layers, an input layer, a minimum of one hidden layer and an output layer. The MLP structure used in this work is shown in Figure 1. The structure was selected based on trial and error. During trial and error, the transfer function, the training function and the number of nodes were varied. It was found that a single hidden layer with 25 nodes, hyperbolic tangent sigmoid function for transfer and quasi-Newton 
backpropagation function for training were sufficient to produce an MLP structures, which could predict the target variable with $\mathrm{R}$ values between $0.97-0.99$.

The inputs in the MLP shown in Figure 1 is a 12 by 1 matrix, which is listed in Table 1. Separate MLP were developed for the target variables shown in Table 1. Therefore, the output in each MLP was a single element.

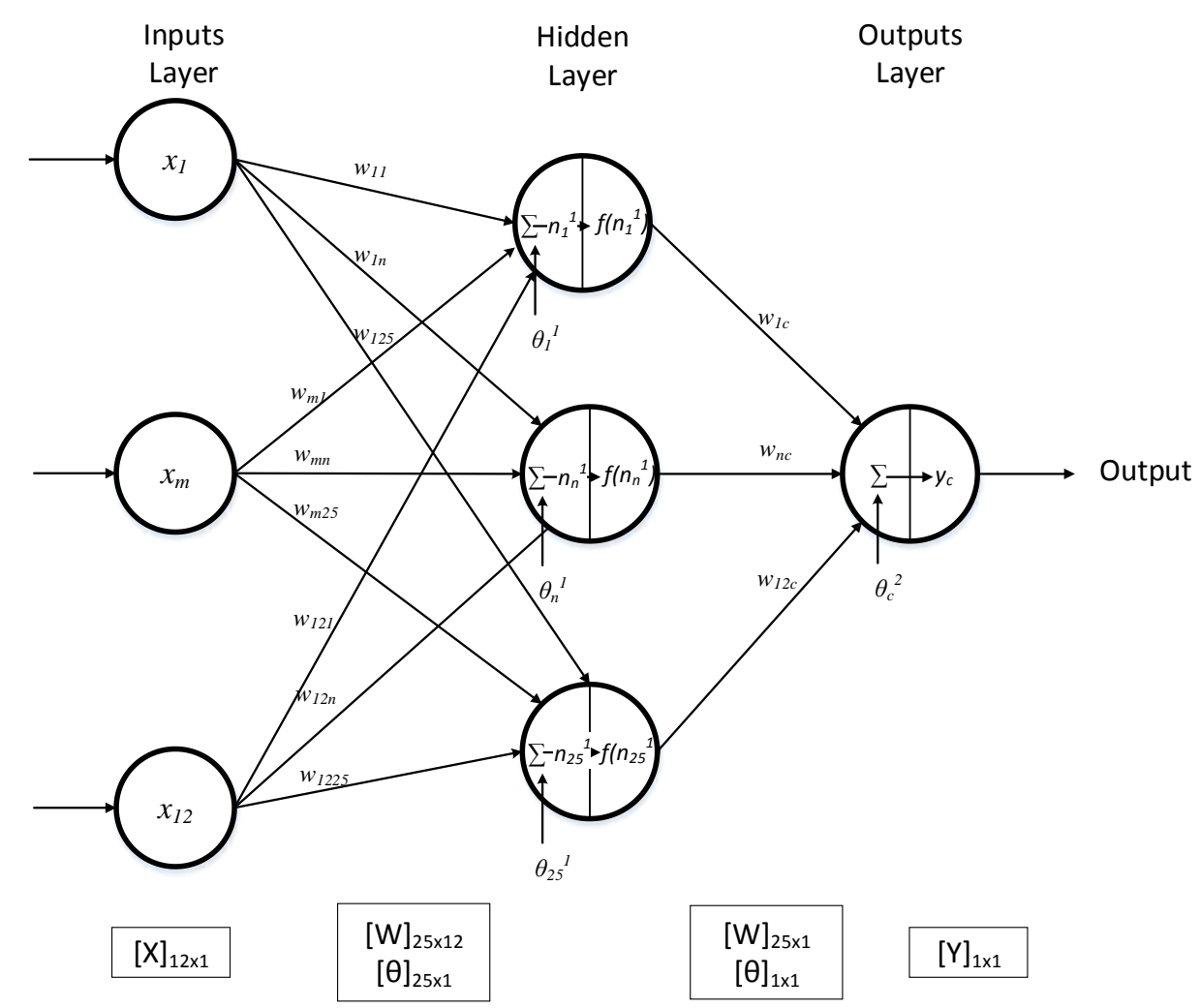

Figure 1: Structure of the MLP used in this study

The steps followed in the prediction of target variables are as follows:

1) The hidden layer receives the weighted input, which is summed up with a constant bias as

$$
n_{i}^{1}=w_{j i} x_{j}+\theta_{i}^{1}
$$


where, $j=1, \ldots, 12, i=1, \ldots, 25, x_{j}$ is input to MLP, $w_{j i}$ are weights, $\theta_{i}$ are the constant bias terms of the hidden layer, $n_{i}^{1}$ is input to the transfer/activation function.

2) Due to the asymptotic nature, hyperbolic tangent sigmoid is preferred as transfer function:

$$
f\left(n_{i}^{1}\right)=\frac{e^{n_{i}^{1}}-e^{-n_{i}^{1}}}{e^{n_{i}^{1}}+e^{-n_{i}^{1}}}
$$

The function is applied at all the nodes in the hidden layer.

3) Linear function was used as the transfer function at the output layer. The function computes the output of MLP by summing up weighted output from the hidden layer with a constant bias as

$$
y_{c}=w_{i c} f\left(n_{i}^{1}\right)+\theta_{c}^{2}
$$

where, $i=1, \ldots, 25, f\left(n_{i}^{1}\right)$ is output from hidden layer, $n_{i}^{1}$ is input to the transfer/activation function, $w_{i c}$ are weights and $\theta_{c}^{2}$ is the constant bias terms of the output layer.

4) All the computed $y_{c}$ values from the MLP are compared with actual $y_{t}$ values from the training data set using mean square error given as

$$
m s e=\frac{1}{N} \sum_{i=1}^{N}\left(y_{t}-y_{c}\right)_{i}^{2}
$$

where, $\mathrm{N}$ is the size of data set $=444, y_{t}$ is targeted value and $y_{c}$ is computed value. The weights in the hidden layer and the output layer were varied using quasi-Newton backpropagation function until the mse value reaches less than $10^{-4}$ for $\mathrm{G}_{\mathrm{mm}}$ and $10^{-1}$ for the rest of the variables or the number of epochs reaches 2000.

Following the above steps, the overall network can be mathematically expressed as

$$
y_{c}=\sum_{i=1}^{25} w_{i c}\left(\sum_{j=1}^{12} f\left(w_{j i} x_{j}+\theta_{i}^{1}\right)\right)+\theta_{c}^{2}
$$


Optimizing asphalt mix design process using artificial neural network and genetic algorithm

where, $j=1, \ldots, 12, i=1, \ldots, 25, x_{j}$ is input to MLP, $w_{j i}$ are weights, $\theta_{i}^{1}$ are the constant bias terms of the hidden layer, $w_{i c}$ are weights and $\theta_{c}^{2}$ is the constant bias terms of the output layer. Eighty percent of the dataset was used in the neural network training following the steps described above. Ten percent of the dataset was used in validation and another ten percent in testing the trained ANN.

The training, validation and testing of all the ANN models were performed using Matlab's neural network tool box. Comparison of the ANN predicted with laboratory measured values is presented in Figure 2.

As mentioned earlier, initially $\mathrm{G}_{\mathrm{mb}}$ was considered as a target variable instead of Va. However, as it can be seen in Figure 2, a better ANN could be developed for Va compared to $G_{m b}$. Therefore, $G_{m b}$ was computed using $A N N$ obtained $G_{m m}$ and Va. Next, the accuracy of the ANN models were evaluated at typically accepted tolerance values. It was found that the ANN models were able to successfully predict $\mathrm{G}_{\mathrm{mm}}$, Va, Stability and Flow for $99.8 \%, 96.0 \%, 97.8 \%$ and $99.1 \%$ of times at acceptable tolerance values of $\pm 0.05, \pm 0.5, \pm 2.0$ and \pm 0.5 , respectively. On reducing the acceptable tolerance values by half, the success percentage for $G_{m m}, V a$, stability and flow were $99.6 \%, 74.1 \%, 87.8 \%$ and $95.5 \%$, respectively.

The performance of ANN models shows that the proposed MLP structure was able to learn and predict the targeted variables within acceptable error. Therefore, the ANN models were found to be well suited in developing optimization tool to aid asphalt mix design. 

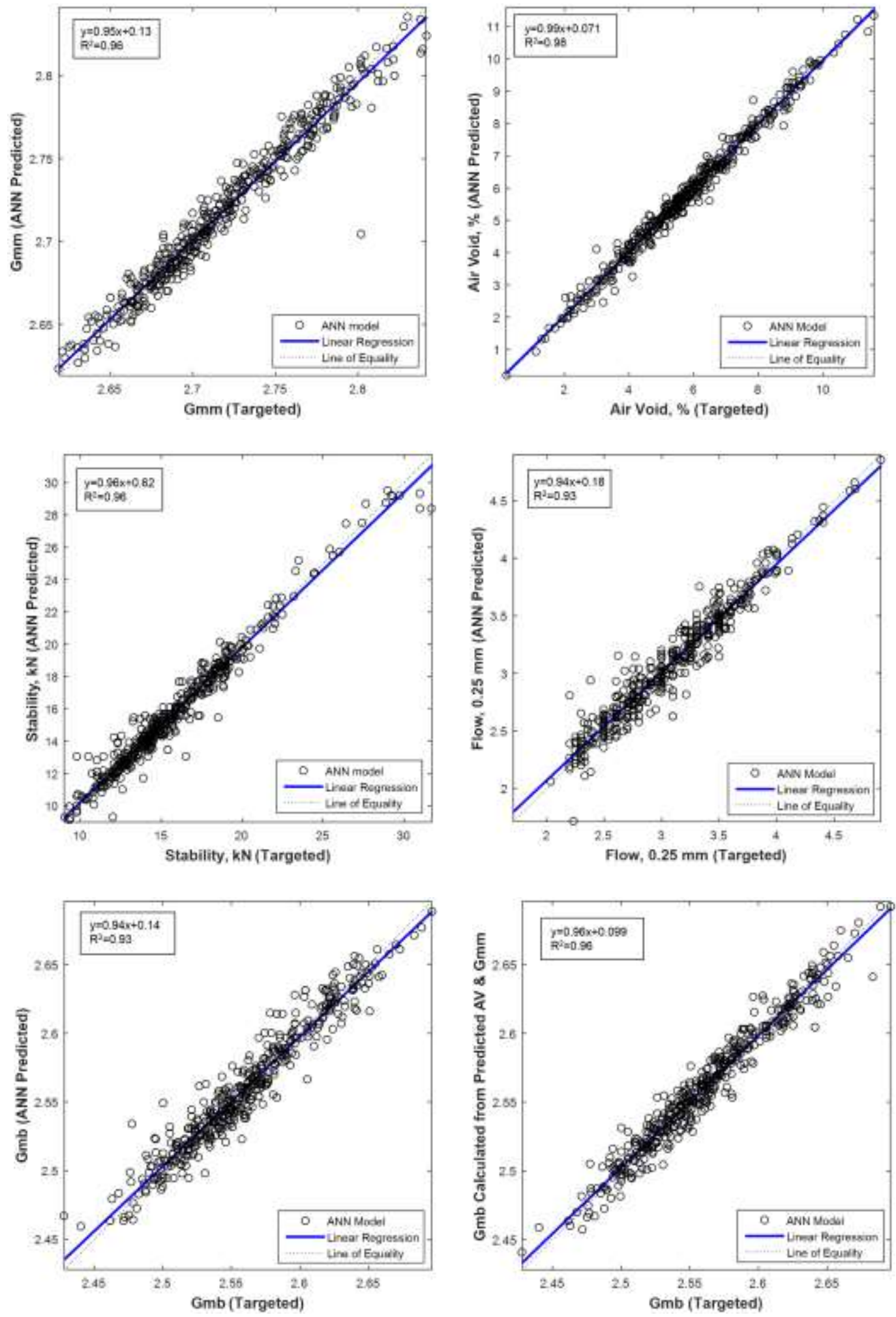

Figure 2: Comparison of ANN predicted versus measured values for $G_{\mathrm{mm}}$, Va, stability, flow and $G_{\mathrm{mb}}$ 
Optimizing asphalt mix design process using artificial neural network and genetic algorithm

\section{GA Model to Optimize Asphalt Mix}

Traditional optimization techniques used in pavement engineering are generally based on direct search (such as Hooke-Jeeves, simplex) or gradient search methods (such as secant method, Newton Raphson, modified Powell hybrid and modified Levenberg-Marquardt). In absence of a continuous differentiable function, the traditional optimization techniques are limited in their ability to find a solution. Furthermore, the solution obtained from these techniques are dependent on the location of the starting point in the search. GA have been shown to produce much superior results compared to the traditional techniques. The advantages of GA are that (i) it does not require calculating gradient and (ii) it can produce global solution (Deb, 2009).

GA is a non-traditional optimization technique based on evolutionary algorithm (EA), in which, each iteration moves the problem towards a better solution. A typical GA problem includes an objective function, which is required to be optimized (minimized or maximized), taking into consideration that the solution lies within a search space defined by the limits (bounds) on the variables. First step in a GA optimization problem that does not include nonlinear constraints is to generate initial set of candidate solution called population, which are within the search space. Next all the candidate solutions are ranked based on their objective function value. Out of these candidates, some of the solutions are selected using a probability based scheme and the rest are discarded. The selected candidates are called parents. Crossover and mutation are the two steps in which new candidates called offspring are produced through flow of information from one parent into another and inducing localized changes. Based on termination criteria either these offspring are used as initial pool of solutions to continue to next iteration, or the best among the offspring is chosen as the optimized solution. 
Most of the GA application in pavement engineering are in analysis of either FWD data (Chatti et al., 2017) or maintenance and rehabilitation strategy (Bosurgi and Trifiro, 2005), Panagopoupou and Chassiakos, 2012). GA in FWD data analysis is used to estimate in situ properties of pavement layers by minimizing difference between field-measured and predicted deflections. Varma and Kutay (2013) and Varma and Kutay (2016) developed linear-constrained GA based FWD analysis model called BACKLAVA and BACKLAVAN. The constraints were introduced to reduce search space in their problem formulation. The authors related the sigmoid function curve parameters with linear viscoelastic properties of asphalt mix to propose simple linear constraint in their formulation. However, they showed that the linear constraint can be embedded in the objective function itself by redefining the variables, thus converting the linear constrained GA problem into an unconstrained GA. Alternately, the linear constraints, along with the upper and lower limits can be used to always generate populations that comply with them. However, this is not always possible in a non-linear constrained GA problem.

In general, non-linear constrained optimization problems are solved by penalizing the objective function for any violation to the constraints (Deb, 2000), (Conn et al., 1991). This penalty ensures that the search is systematically dragged away from any search space which is violating non-linear constraints. In this paper Matlab function ' $\mathrm{ga}$ ' is used to perform non-linear constrained optimization. The function ' ga' handles the Non-linear equality and inequality constraints by creating an augmented Lagrangian form called sub-problem for the original problem (Conn et al. 1991), (Conn et al. 1997). Equation 8 shows the augmented Lagrangian form of a general original problem in Equation 7

$$
\min f(x), \text { for } x
$$

such that: 


$$
\begin{gathered}
C_{i}(x) \leq 0, i=1 \text { to } n \\
\operatorname{Ceq}_{j}(x)=0, j=1, m \\
x^{l} \leq x \leq x^{u}
\end{gathered}
$$

where, $f(x)$ is objective function, $n$ and $m$ are number of inequality and equality non-linear constraints, $C_{i}(x)$ and $\mathrm{Ceq}_{j}(x)$ are inequality and equality non-linear constraints

$$
\min \psi(x, \lambda, s, \rho)=f(x)-\sum_{i=1}^{m} \lambda_{i} s_{i} \log \left(s_{i}-C_{i}(x)\right)+\sum_{j=1}^{n} \lambda_{j} \operatorname{Ceq}_{i}(x)+\frac{\rho}{2} \sum_{j=1}^{n} \operatorname{Ceq}_{i}(x)^{2}
$$

Where, $\lambda_{i}$ are non-negative Lagrange multipliers, $s_{i}$ are slack variables. In non-linear constrained GA, this augmented Langragian sub-problem is minimized. In each GA iteration the Langragian coefficients are fixed and the sub-problem thus obtained is minimized until termination criteria reaches. It is worth noting that since the sub-problem strips the non-linear constraints from the original problem, it is optimized using GA with only linear constraints and bounds to be satisfied. Figure 3 shows the procedure followed in obtaining an optimized gradation using the ANN-GA hybrid approach.

\section{Case Study}

The selection of aggregate gradation and binder content affects the cost and quality of asphalt mix. Cost of asphalt mix increases with the use of aggregates with higher specific gravity or high binder content. Increase in specific gravity of aggregates is in general related to increase in size of the largest aggregate in the mix. However, increase in size of the largest aggregate in mix leads to reduced design asphalt content and lower overall mix cost. Therefore, binder is typically considered as the most expensive component of asphalt mix. In the present study, hot bin percentages and binder content are optimized to obtain an asphalt mix that satisfies 
specification requirements at the lowest possible binder content. This means that, the objective function for the case study is to be minimized $\mathrm{P}_{\mathrm{b}}$.

Apart from the cost consideration, another reason for considering binder content minimization as the objective of the case study is that, all the mixes in this study were intended for projects in the subtropical hot desert climate, where the maximum air temperatures in the months of May, June, July and August are $42.6^{\circ} \mathrm{C}, 43.6^{\circ} \mathrm{C}, 45.3^{\circ} \mathrm{C}$ and $44.3^{\circ} \mathrm{C}$, respectively. Therefore, the temperature of pavement at $20 \mathrm{~mm}$ depth in the region has a tendency to go up to about $70^{\circ} \mathrm{C}$. In these hot weather condition, bleeding is one of the major concerns. Naturally, in the regional pavement industry there is a tendency to lower binder content in asphalt mixes to avoid any potential risk of bleeding. Minimizing binder content may end up depriving the aggregates from enough surface film thickness, which can subsequently lead to reduction in durability. To address this issue, the formulated model includes a constraint on minimum $\mathrm{FT}_{\mathrm{b}}$ requirement. Although the binder content is minimized during the GA optimization, the asphalt mix is checked for the $\mathrm{FT}_{\mathrm{b}}$, which is calculated using MS-2 Asphalt Institute (2014) surface area method.

It should be noted that the overall approach developed here is general and the objective function can be replaced by any other expression that requires optimization (e.g. stability or density or a combination). Formulation of the optimization model using GA is:

$$
\min P_{b}
$$

such that: Limits on GA variables:

$$
\begin{gathered}
H B_{k}^{l}+H B_{k}^{m} \leq H B_{k} \leq H B_{k}^{u}-H B_{k}{ }^{m} ; \\
P_{b}^{l}+P_{b}^{m} \leq P_{b} \leq P_{b}^{u}-P_{b}^{m}
\end{gathered}
$$




$$
\begin{gathered}
\text { linear Constraints: } \\
P_{d}^{l}+P_{d}^{m} \leq P_{d} \leq P_{d}{ }^{u}-P_{d}^{m} ; \\
F T_{b}{ }^{l} \geq 8 \\
H B_{1}=100-\sum_{k=2}^{n} H B_{k} \\
N o n-l i n e a r \text { Constraints: } \\
S \geq S^{u}+S^{m} ; \\
F^{l}+F^{M} \leq F \leq F^{u}-F^{m} ; \\
V a^{l}+V a^{m} \leq V a \leq V a^{u}-V a^{m} ; \\
V M A-V M A^{m} \geq 14 ; \\
V F B^{l}+V F B^{m} \leq V F B \leq V F B^{u}-V F B^{m} ; \\
F B R^{l}+F B R^{m} \leq F B R \leq F B R^{u}-F B R^{m}
\end{gathered}
$$

where, $H B_{k}$ is percentage of hot bin size $k=2$ to $n$ where $n$ is number of hot bins; $P_{b}$ is percent bitumen by weight; $P_{d}$ is percent passing sieve size $d=25.0 \mathrm{~mm}, 19.0 \mathrm{~mm}, 12.5 \mathrm{~mm}, 9.5 \mathrm{~mm}$, $4.75 \mathrm{~mm}, 2.36 \mathrm{~mm}, 1.18 \mathrm{~mm}, 0.425 \mathrm{~mm}$ and $0.075 \mathrm{~mm} ; F T_{b}$ is bitumen film thickness, calculated using MS-2 Asphalt Institute method; $S$ is stability, obtained from ANN model, $F$ is flow, obtained from ANN model; $V a$ is obtained from ANN model; $V M A$ is calculated using Equation 10; VFB is calculated using Equation 11; FBR is calculated using Equation 12; upper script $l$ denotes lower limit; upper script $u$ denotes upper limit; upper script $m$ denotes marginal limit. 


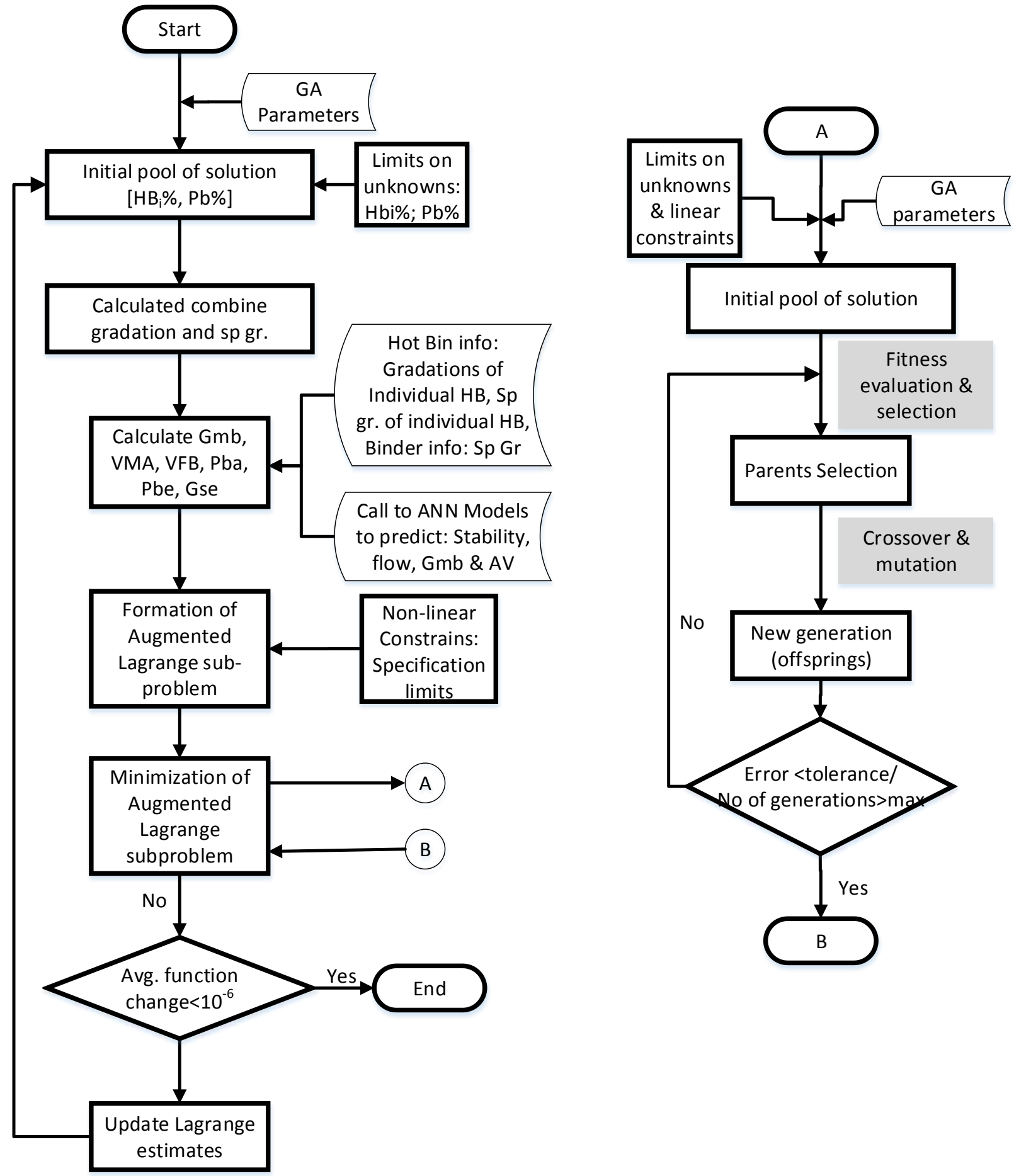

Figure 3: Flow chart for the ANN-GA approach using non-linear constrained optimization

$$
V M A=\left(100-\frac{G_{m b}}{G_{s b}}\left(100-P_{b}\right)\right)
$$


where, $G_{m b}$ and $G_{s b}$ are calculated using Equation 13 and Equation 1 respectively.

$$
\begin{aligned}
& V F B=\frac{(V M A-V a)}{V M A} 100 \\
& F B R=\frac{P_{0.075}}{P_{b e}}
\end{aligned}
$$

where, $P_{0.075}$ is percent passing sieve $0.075 \mathrm{~mm} ; P_{b e}$ is effective binder content of the mix.

$$
G_{m b}=\left(1-\frac{V a}{100}\right) G_{m m}
$$

where, $V a$ and $G_{m m}$ are both obtained from ANN models.

The non-linear constraints in Equation 9 shall not be confused with limits on variables namely $H B_{k}$ and $P_{b}$. The values for all the parameters in the non-linear constraints are either obtained from ANN models or derived using them. Therefore, they are not directly related to the actual variables of the GA, $H B_{k}$ and $P_{b}$. In fact, although the expressions in non-linear constraints appear to be linear, they are obtained through non-linear mapping of variables $H B_{k}$ and $P_{b}$ in ANN (through Equation 6). The developed model is compiled into a computer software called AMO that can be used by transportation agencies as a mix design tool.

The addition of marginal limits are often considered in field for quality control purpose. This is included in the formulation only to provide an option for designers to gain confidence that the selected gradation is not too close to upper or lower limits in specifications.

\section{Laboratory Verification of the Proposed Approach}

A laboratory testing plan was implemented to verify the approach developed in this paper. The objectives of the laboratory study was to check the following: (i) performance of the ANN model close to the range of the data set that were used for the development of the model; (ii) compliance 
Optimizing asphalt mix design process using artificial neural network and genetic algorithm

of ANN model to fundamental requirements and (iii) performance of the ANN-GA approach in obtaining an optimized gradation. For the laboratory testing, fresh hot bin aggregates and polymer modified PG 76-10 binder were obtained from asphalt plant. The properties of the hot bin aggregates are shown in Table 2.

Five job mix formula (JMF) were generated using the hot bins shown in Table 2. The first three mixes were generated to check performance of the developed ANN models. JMFs of these three mixes were obtained from construction projects. Hot bins percentages were adjusted to obtain the gradations. By comparing Table 1 and Table 2 it can be seen that for the first mix, Mix-1, the gradation was outside the JMFs that were used to develop the ANN models. Whereas, the JMFs of Mix-2 and Mix-3 were within the range of the JMFs database for the ANN models. Next, the optimization approach proposed in the study was used to obtain mixes that satisfy the specification requirements presented in Table 3 at the lowest possible binder content. At first, the GA optimization was performed at population-generation combination of 50-5, without considering the film thickness constraint. The gradation and binder content outputted from the first run is referred in the paper as Mix-4. The film thickness for Mix-4 was found to be 7.9 microns. Since the film thickness in Mix-4 was less than 8.0 microns it was rejected. Next, the GA optimization was performed at population-generation combination of 200-5. The gradation and binder content output from the second run is referred in the paper as Mix-5. Film thickness for Mix-5 was found to be 8.2 microns. 
Optimizing asphalt mix design process using artificial neural network and genetic algorithm

TABLE 2: Job mix formula of asphalt mixed used in laboratory verification

\begin{tabular}{|c|c|c|c|c|c|c|c|c|c|c|c|}
\hline \multicolumn{2}{|c|}{ Property } & \multicolumn{5}{|c|}{ Hot Bin Gradation } & & \multicolumn{4}{|c|}{ Combined Gradation } \\
\hline Parameter & $\begin{array}{c}\text { Sieve } \\
\text { Size } \\
\mathrm{mm}\end{array}$ & $\begin{array}{c}\text { HB1- } \\
22-12 \\
\mathrm{~mm}\end{array}$ & $\begin{array}{c}\mathrm{HB} 2- \\
12-7 \\
\mathrm{~mm}\end{array}$ & $\begin{array}{c}\text { HB3- } \\
7-4 \\
\text { mm }\end{array}$ & $\begin{array}{c}\text { HB4- } \\
4-0 \\
\text { mm }\end{array}$ & $\begin{array}{l}\text { HB5- } \\
\text { Filler }\end{array}$ & Mix-1* & Mix-2 & Mix-3 & Mix-4 & Mix-5 \\
\hline \multirow{9}{*}{$\begin{array}{c}\text { Percent } \\
\text { Passing } \\
(\%)\end{array}$} & 25.0 & 100 & 100 & 100 & 100 & 100 & 100 & 100 & 100 & 100 & 100 \\
\hline & 19.0 & 89 & 100 & 100 & 100 & 100 & 100 & 100 & 98 & 98 & 98 \\
\hline & 12.5 & 13 & 100 & 100 & 100 & 100 & 88 & 81 & 83 & 81 & 81 \\
\hline & 9.5 & 1.1 & 62 & 100 & 100 & 100 & 75 & 69 & 70 & 71 & 71 \\
\hline & 4.75 & 0.7 & 1.1 & 66 & 98 & 100 & 46 & 46 & 47 & 51 & 53 \\
\hline & 2.36 & 0.6 & 0.7 & 3 & 82 & 100 & 29 & 31 & 31 & 33 & 38 \\
\hline & 1.18 & 0.6 & 0.6 & 1.0 & 55 & 100 & 19 & 18 & 21 & 23 & 26 \\
\hline & 0.425 & 0.6 & 0.6 & 1.0 & 28 & 100 & 11 & 12 & 12 & 13 & 15 \\
\hline & 0.075 & 0.4 & 0.5 & 1.0 & 6.0 & 81 & 4.8 & 4.6 & 4.6 & 4.6 & 5.0 \\
\hline $\mathrm{G}_{\mathrm{sb}}$ & 2.943 & 2.906 & 2.877 & 2.824 & 2.824 & 2.943 & 2.877 & 2.879 & 2.878 & 2.876 & 2.873 \\
\hline $\mathrm{P}_{\mathrm{b}} \%$ & na & na & na & na & na & na & 4.3 & 4.0 & 4.1 & 3.91 & 3.96 \\
\hline $\begin{array}{c}\text { PG } \\
\text { Grade }\end{array}$ & na & na & na & na & na & na & $\begin{array}{c}\text { PG } \\
76-10\end{array}$ & $\begin{array}{c}\text { PG } \\
76-10\end{array}$ & $\begin{array}{c}\text { PG } \\
76-10\end{array}$ & $\begin{array}{c}\text { PG } \\
76-10\end{array}$ & $\begin{array}{c}\text { PG } \\
76-10\end{array}$ \\
\hline
\end{tabular}

*Mix-1 gradation outside the JMFs in the data base used to develop the ANN models

TABLE 3: Boundary and marginal limits on variables and constraints in GA optimization

\begin{tabular}{|c|l|c|c|c|}
\hline Variables & \multicolumn{1}{|c|}{ Parameter } & $\begin{array}{c}\text { Lower } \\
\text { Limit }\end{array}$ & $\begin{array}{c}\text { Upper } \\
\text { Limit }\end{array}$ & $\begin{array}{c}\text { Marginal } \\
\text { Limit }\end{array}$ \\
\hline \multirow{4}{*}{$\begin{array}{c}\text { GA } \\
\text { Variables }\end{array}$} & Hot Bin \%, HB2 & 5 & 50 & 0 \\
\cline { 2 - 5 } & Hot Bin \%, HB3 & 5 & 50 & 0 \\
\cline { 2 - 5 } & Hot Bin \%, HB4 & 5 & 50 & 0 \\
\cline { 2 - 5 } & Hot Bin \%, HB5 & 0 & 7 & 0 \\
\cline { 2 - 5 } & Binder Content Pb & 3.5 & 4.5 & 0.2 \\
\hline \multirow{5}{*}{$\begin{array}{c}\text { Constraint } \\
\text { Parameters }\end{array}$} & \% passing 19.0 mm & 80 & 100 & 4 \\
\cline { 2 - 5 } & \% passing 12.0 mm & 63 & 85 & 4 \\
\cline { 2 - 5 } & \% passing 9.5 mm & 57 & 77 & 4 \\
\cline { 2 - 5 } & \% passing 4.75 mm & 40 & 60 & 3 \\
\cline { 2 - 5 } & \% passing 2.36 mm & 25 & 45 & 3 \\
\cline { 2 - 5 } & \% passing 0.425 mm & 10 & 22 & 2 \\
\cline { 2 - 5 } & Stability, S (kN) & 2 & 8 & 1 \\
\cline { 2 - 5 } & Flow, F (0.25 mm) & 2.5 & 4.0 & 0 \\
\cline { 2 - 5 } & Air Void, Va (\%) & 4.5 & 8 & 1 \\
\cline { 2 - 5 } & $\begin{array}{l}\text { Bitumen film thickness } \\
\text { (FT } \text { b microns }\end{array}$ & 8.0 & - & - \\
\cline { 2 - 5 } & VMA (\%) & 14 & - & - \\
\cline { 2 - 5 } & VFB (\%) & 50 & 75 & 0 \\
\cline { 2 - 5 } & $\begin{array}{l}\text { Filler to binder ratio } \\
\text { (FBR) }\end{array}$ & 0.8 & 1.5 & 0 \\
\hline & & & & \\
\hline
\end{tabular}


To accept the prediction of asphalt mix properties it is mandatory that the solution complies with the following three fundamental rules:

$$
\begin{aligned}
& G_{m m} \nless G_{m b} \\
& G_{s e} \nless G_{s b} \\
& P_{b a} \nless 0
\end{aligned}
$$

Compliance of these fundamental rules can be viewed as protection to ensure the results are meaningful. It can be seen in Table 4 that all the three fundamental rules are achieved in the ANN model. Therefore, the predicted results can be considered eligible to compare with laboratory obtained results. Asphalt mixes Mix-1, Mix-2, Mix-3 and Mix-5 were tested in laboratory to verify the ANN predicted results.

TABLE 4: Fundamental properties of asphalt mix obtained from ANN

\begin{tabular}{|c|c|c|c|c|c|}
\hline Parameter & Mix-1 & Mix-2 & Mix-3 & Mix-4 & Mix-5 \\
\hline $\mathrm{G}_{\mathrm{mm}}$ & 2.700 & 2.718 & 2.712 & 2.709 & 2.703 \\
\hline $\mathrm{G}_{\mathrm{mb}}$ & 2.517 & 2.554 & 2.539 & 2.519 & 2.518 \\
\hline $\mathrm{G}_{\mathrm{se}}$ & 2.911 & 2.916 & 2.914 & 2.900 & 2.898 \\
\hline $\mathrm{P}_{\mathrm{ba}}, \%$ & 0.42 & 0.46 & 0.44 & 0.30 & 0.31 \\
\hline
\end{tabular}

Figure 4 presents comparison of asphalt mix volumetric properties obtained from the GA-ANN model, AMO, with laboratory measurements. It can be seen from the figure that the model was able to estimate asphalt properties for the Mix-2, Mix-3 and Mix-5 within the laboratory precision limit prescribed in ASTM. As expected, some of the parameters predicted by ANN for Mix-1 did not match laboratory measurements. This is expected, as it is well known that ANN models cannot accurately predict inputs that are outside the range of the data that were used during the training of the ANN networks. 

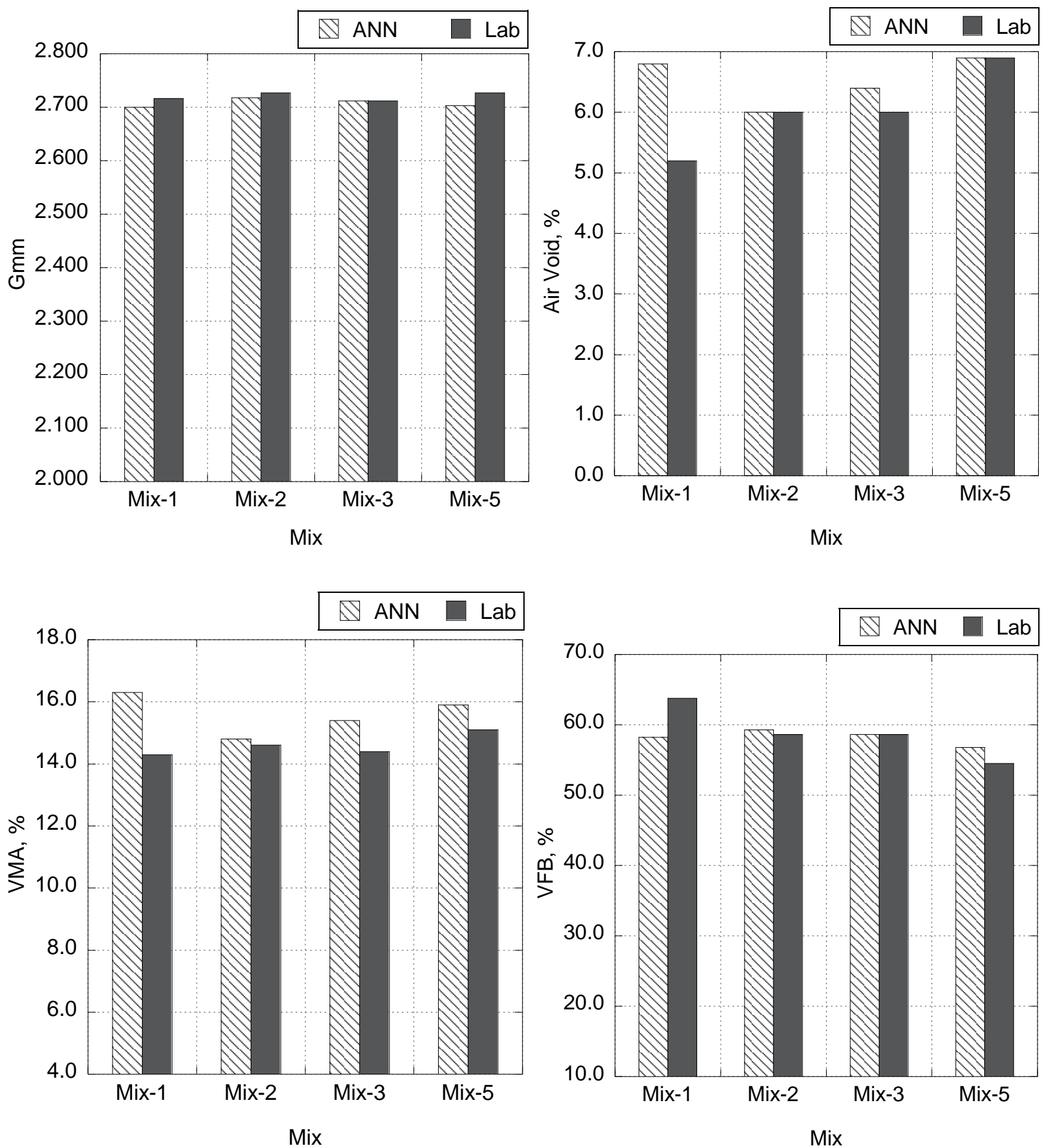

*Mix-1 gradation outside the JMFs in the data base used to develop the ANN models

Figure 4: Comparison of the laboratory measured results with ANN model prediction 


\section{SUMMARY AND CONCLUSION}

In this study an ANN and GA based optimization model called AMO has been developed to automate selection of aggregate gradation and binder content to produce asphalt mixtures that comply with applicable specification requirements. Successful implementation of the approach was demonstrated for Marshall mixes.

The model is developed using feed forward backpropagation neural network with a single hidden layer MLP structure. The input variables in the model were aggregate gradation, percent binder content, combine bulk specific gravity of aggregate and PG grade high temperature. It was found that hidden layer with 25 nodes, hyperbolic tangent sigmoid function for transfer and quasiNewton backpropagation function for training were sufficient to produce $G_{m m}, V a$, stability and flow values with $R$ values between $0.97-0.99$. The model was able to successfully predict $\mathrm{G}_{\mathrm{mm}}$ $( \pm 0.025), \mathrm{Va}( \pm 0.5 \%)$, stability $( \pm 2 \mathrm{kN})$ and flow $( \pm 0.25 \mathrm{~mm})$ for $99.6 \%, 96.0 \%, 97.8 \%$ and $95.5 \%$ of the time, respectively. Further, it was found that the ANNs produce results such that $G_{m m}>$ $G_{m b}, G_{s e}>G_{s b}$ and $P_{b a}>0$, indicating that the predicted results are fundamentally meaningful.

The developed ANN models were then called in a non-linear constrained GA to optimize aggregate gradation. The Marshall requirements were put as non-linear constraints in the optimization formulation and the binder content was minimized. The non-linear constraints were handled by using augmented Langragian algorithm. Four new asphalt mixes different from the ANN database were prepared in laboratory using hot bin aggregates and polymer modified binder obtained from an asphalt plant to verify the developed model. It was found that the ANN models were not able to predict Marshall properties of Mix-1. This was expected because, the JMF selected for Mix-1 was intentionally selected outside the range of database that was used to 
Optimizing asphalt mix design process using artificial neural network and genetic algorithm

train the ANN models. Comparison of ANN predicted and laboratory measured results show that the ANN could predict Marshall properties within the ASTM precision limits.

The model developed in this paper should be considered more conceptual. Although the approach is demonstrated for Marshall compacted mixes, it can be applied to any other mix design method.

\section{ACKNOWLEDGEMENTS}

This study was partially supported by Doha Technical Laboratories (DTL). We thank Ayman Al Ghbani and Ahmed Hindi from DTL, who provided insight and expertise that greatly assisted the research. The incentive funding for rated researchers received from the National Research Foundation (NRF) in South Africa is also gratefully acknowledged.

\section{REFERENCE}

1. Asphalt Institute. (2014), Asphalt Mix Design Methods, MS-2, 7th edition, Asphalt Institute.

2. Birgisson, B. \& Ruth B. (2001), Development of Tentative Guidelines for the Selection of Aggregate Gradations for Hot-Mix Asphalt. Aggregate Contribution to HMA Performance, ASTM 1412, 2001.

3. Bosurgi, G. \& Trifiro, F. (2005), A model based on artificial neural networks and genetic algorithms for pavement maintenance management. International Journal of Pavement Engineering. Vol 6, pp. 201-209.

4. Chatti, K., Kutay, E., Lajnef, N., Zaabar, I., Varma, S. \& Lee, S. (2014), Enhanced Analysis of Falling Weight Deflectometer Data for use with Mechanistic-Empirical Flexible 
Optimizing asphalt mix design process using artificial neural network and genetic algorithm

Pavement Design and Analysis and Recommendations for Improvements to Falling Weight

Deflectometer. FHWA Report, Washington, DC.

5. Choi, J., Adams, T., \& Bahia, H. (2004), Pavement roughness modeling using backpropagation neural networks. Computer-Aided Civil and Infrastructure Engineering, 19(4), pp. 295-303.

6. Commuri, S., Mai, A. \& Zaman, M. (2011), Neural network based intelligent compaction analyzer for estimating compaction quality of hot mix asphalts. Journal of Construction Engineering and Management, 137 (9), pp. 634-644.

7. Conn, A., Gould, N. \& Toint, L. (1997), Globally Convergent Augmented Lagrangian Barrier Algorithm for Optimization with General Inequality Constraints and Simple Bounds. Mathematics of Computation, Vol 66 (217), pp. 261-288.

8. Conn, A., Gould, N. \& Toint, L. (1991), Globally Convergent Augmented Lagrangian Algorithm for Optimization with General Constraints and Simple Bounds. SIAM Journal on Numerical Analysis, Vol 28(2), pp. 545-572.

9. Deb, K. (2000), An efficient constraint handling method for genetic algorithms," Computer Methods in Applied Mechanics and Engineering, 186 (2-4), pp. 311-338.

10. Deb, K. (2009), Optimization for Engineering Design: Algorithms and examples. PHI, New Delhi.

11. Far, M., Underwood, B., Ranjithan, S., Kim, Y. \& Jackson, N. (2009), Application of Artificial Neural Networks for Estimating Dynamic Modulus of Asphalt Concrete. Transportation Research Record, 2127, pp. 173-186.

12. Fuller, W.B. and Thompson, S. (1907), The laws of proportioning concrete, Transactions of the ASCE, v. 159. 
Optimizing asphalt mix design process using artificial neural network and genetic algorithm

13. Gopalakrishnan, K., Kim, S., Ceylan, H. \& Kaya, O. (2014), Development of asphalt dynamic modulus master curve using falling weight deflectometer measurements. Ames, IA: Iowa DOT, Final Report.

14. Huang, Y. \& Moore, R. (1997), Roughness level probability prediction using artificial neural networks," Transportation Research Record 1592, pp. 89-97.

15. Krcmarik, M., Varma, S., Kutay, E. \& Jamrah, A. (2016), Development of Predictive Models for Low-Temperature Indirect Tensile Strength of Asphalt Mixtures. Journal of Materials in Civil Engineering, 28(11).

16. Li, Y. \& Wang L. (2015), Computer-aided procedure for determination of asphalt content in asphalt mixture using discrete element method. International Journal of Pavement Engineering, pp. 1-10.

17. Li, M. \& Wang, H. (2017), Development of ANN-GA program for backcalculation of pavement moduli under FWD testing with viscoelastic and nonlinear parameters," International Journal of Pavement Engineering, pp. 1-9.

18. Lytton, R.L., Tsai F., Lee, S., Luo, R., Hu, S. \& Zhou, F. (2010), Models for predicting reflection cracking of Hot-mix Asphalt Overlays. NCHRP Report 669, Appendix A.

19. McLeod, N. \& Davidson, J. (1981), Particle Index Evaluation of Aggregates for Asphalt Paving Mixtures, Proceedings, Association of Asphalt Paving Technologists, Vol. 50, pp. 251-290.

20. Ozturk, H. \& Kutay, M. (2014), An artificial neural network model for virtual Superpave asphalt mixture design. International Journal of Pavement Engineering, 15(2), pp. 151162. 
Optimizing asphalt mix design process using artificial neural network and genetic algorithm

21. Panagopoupou M. \& Chassiakos A. (2012), Optimization model for pavement maintenance planning and resource allocation. Transportation Research Circular E-C136.

22. Roberts, F., Kandhal, P., Brown, E., Lee, D. \& Kennedy, T. (1996), Hot Mix Asphalt Materials, Mixture Design, and Construction. National Asphalt Paving Association Education Foundation. Lanham, MD.

23. Roque, R., Birgisson, B., Kim, S. \& Gaurin A. (2006), Development of Mix Design Guidelines for Improved Performance of Asphalt Mixtures. Final report, FDOT.

24. Shen, S. \& Yu, H. (2011), Analysis of aggregate gradation and packing for easy estimation of hot-mix-asphalt voids in mineral aggregate. Journal of Materials in Civil Engineering, 23(5), pp. 664-672.

25. Varma, S. \& Kutay, E. (2016), Backcalculation of Viscoelastic and Nonlinear Flexible Pavement Layer Properties from Falling Weight Deflections. International Journal of Pavement Engineering, Vol. 17(5), pp. 388-402.

26. Varma, S., Kutay, E. \& Chatti, K. (2013), Data Requirements from Falling Weight Deflectometer Tests for Accurate Backcalculation of Dynamic Modulus Master curve of Asphalt Pavements. Airfield and Highway Pavement 2013: Sustainability and efficient pavements, California, pp. 445-455.

27. Vavrik, W., Huber, G., Pine, W., Carpenter, S. \& Bailey, R. (2002), Method for Gradation Selection in Hot-Mix Asphalt Mixture Design. Transportation Research E-Circular, Report No: E-C044.

28. Yang, J., Lu, J., Gunaratne, M. \& Xiang, Q. (2003), Forecasting Overall Pavement Condition with Neural Networks: Application on Florida Highway Network. Transportation Research Record, 1853, pp. 3-12. 